\title{
Seismoelectric wave propagation modeling for typical laboratory configurations: A numerical validation
}

Niels Grobbe*, Jürg Hunziker and Evert Slob, Delft University of Technology, Department of Geoscience \& Engineering

\section{SUMMARY}

The seismoelectric effect can be of importance for hydrocarbon exploration as it is complementary to conventional seismics. Besides enabling seismic resolution and electromagnetic sensitivity at the same time, the seismoelectric method can also provide us with additional, high-value information like porosity and permeability. However, very little is still understood of this complex physical phenomenon. Therefore, it is crucial to be able to perform numerical modeling experiments to carefully investigate the effect and the parameters that play a role. Over the last couple of years, several seismoelectric laboratory experiments have been carried out in an attempt to validate the underlying theory of the phenomenon and to better understand this complex physical phenomenon. We have recently extended our analytically based, numerical seismoelectric modeling code 'ESSEMOD' to be able to model seismoelectric wave propagation in arbitrarily layered Earth geometries with fluid / porous medium / (fluid) interfaces. In this way, we are capable of effectively simulating full seismoelectric wave propagation, i.e. all existing seismoelectric and electroseismic source-receiver combinations, in typical laboratory configurations. We present the underlying theory that is required for the extension towards arbitrary fluid / porous medium / (fluid) geometries and an effective way to incorporate this in a general seismoelectric layered Earth modeling code. We then validate the underlying global reflection scheme by comparing it with an independently developed layered Earth modeling code for purely electromagnetic fields. The results show a perfect match in both amplitude and phase, indicating that ESSEMOD is correctly modeling the electromagnetic parts of the seismoelectric wave propagation in horizontally layered media with fluid / porous medium / fluid transitions. We finalize with a seismoelectric reciprocal modeling experiment, proving that also the full seismoelectric wave propagation through fluid / porous medium transitions is modeled consistently.

\section{INTRODUCTION}

The field of geophysics focuses on imaging the subsurface of the Earth and extracting pertinent information regarding dynamic processes. As the easily accessible natural resources are running out, there is a continuous demand for improved imaging methods.

In recent years, some research has been performed on seismoelectric phenomena, where coupled seismic waves and electromagnetic fields are considered together (e.g, Pride (1994), Haartsen and Pride (1997), Revil et al. (2007)). The seismoelectric effect can be of importance for hydrocarbon exploration as it is complementary to conventional seismics (e.g., Thompson and Gist (1993), Schoemaker et al. (2012)). Seismoelectric fields can provide us with both seismic (Biot's poroelasticity equations) and electromagnetic (Maxwell's electro- magnetic equations) sensitivities that are coupled to each other via a coupling coefficient (Pride (1994), Pride and Haartsen (1996)). This coupling coefficient can provide us with important supplemental information about for example the permeability or porosity of the medium, and its pore-fluid content.

The main reason why seismoelectric techniques are not yet widely applied in industry, is that the seismoelectric conversion has a very low signal-to-noise ratio. In addition, very little is still understood of this complex physical phenomenon. Therefore, it is crucial to be able to perform numerical modeling experiments to carefully investigate the effect and the parameters that play a role. However, to really make a step towards successful application of seismoelectric methods in the field, the numerical modeling results have to be compared and validated using laboratory experiments.

Over the last couple of years, several seismoelectric laboratory experiments have been carried out in an attempt to validate the posed theory by Pride (1994) and to better understand this complex physical phenomenon (e.g., Zhu et al. (2000), Zhu and Toksöz (2005), Schoemaker et al. (2012)). Typically, the laboratory experiments are carried out using water-tanks in which the sources, receivers and sample under consideration can be deployed. In order to successfully model these kinds of configurations, the numerical algorithm must be able to handle configurations with fluid / porous medium interfaces correctly. Some recent examples of comparisons between laboratory measurements and theoretical numerical predictions are Schakel et al. (2011) and Smeulders et al. (2014). However, the numerical modeling scheme used in these articles was specifically designed for the laboratory configuration at use. The schemes make use of an explicitly defined seismoelectric reflection coefficient at a certain interface (Schakel and Smeulders, 2010). Furthermore, only the electric field components due to an acoustic pressure source could be modeled, thereby only considering the seismoelectric P-SV-TM propagation mode.

Over the last years, we have developed 'ESSEMOD', an analytically based, numerical modelling code for ElectroSeismic and SeismoElectric wave propagation modelling in layered media (Grobbe et al. (2012), Grobbe and Slob (2013)). It is set-up in a similar way as other existing seismoelectric layered Earth codes (Haartsen and Pride (1997), Garambois and Dietrich (2002)) However, 'ESSEMOD' is capable of modeling all existing energy flux-normalized seismoelectric sourcereceiver combinations. It makes use of a global reflection scheme, which leads to an efficient numerical scheme due to the fact that explicit calculation of the scattering matrices at each interface is not required.

We have recently extended 'ESSEMOD' to be able to model seismoelectric wave propagation in arbitrarily layered Earth geometries with fluid / porous medium / (fluid) interfaces. In this way, we are capable of effectively simulating full seis- 
moelectric wave propagation (i.e. all existing seismoelectric source-receiver combinations) in typical laboratory configurations, meaning a porous sample in a water tank.

After discussing shortly the underlying theory that is required for the extension towards fluid / porous media geometries, we will present a first aspect of the actual validation of the numerical code itself: the comparison with an independently developed layered Earth modeling code for purely electromagnetic fields (Hunziker et al., 2014). By putting the coupling coefficient in our modeling code to zero, the poroelastic system completely decouples from the electromagnetic system. In this way, 'ESSEMOD' can also be used to model poroelastic and electromagnetic wave phenomena in layered Earth models. In addition, the decoupled system provides us with the means to reduce the complexity of the recordings, thereby enabling to validate specific parts of the algorithm, in this case the reflection and transmission at fluid / porous media interfaces. We will finalize with a seismoelectric reciprocal modeling experiment in a geometry with fluid / porous medium transitions.

\section{THEORY}

The underlying theory of the numerical algorithm of 'ESSEMOD', is based on the governing equations as derived by Pride (1994). Following Haartsen and Pride (1997), we have split the system of equations into two seismoelectric propagation modes, the SH-TE mode and the P-SV-TM mode. In the SHTE mode, horizontally polarized shear waves are coupled to transverse electric fields. The P-SV-TM mode describes the coupling of pressure waves, via vertically polarized shear waves, to transverse magnetic fields. These two modes are treated independently as one-way wavefields throughout the algorithm, and finally combined again into the full two-way wavefields at the receiver level.

Compared to the theory as described in Haartsen and Pride (1997), we introduce some changes. Since we prefer the system to be energy flux-normalized, we have reorganized the system of equations in such a way that we obtain an optimal symmetry to apply the energy flux-normalization (see for details e.g. Ursin (1983)). Furthermore, we use particle velocity fields instead of displacement by introducing an additional time derivative to the system. In addition, we want the modeling code to be able to independently model poroelastic and electromagnetic phenomena, when the coupling coefficient is set to zero. Namely, the resulting decoupled poroelastic and electromagnetic systems provide us with the means to reduce the complexity of the recordings, thereby enabling to validate and debug specific parts of the algorithm. Our initial modeling results showed that the system as used by Haartsen and Pride (1997) or equivalently Garambois and Dietrich (2002) incorrectly models situations where the coupling coefficient between the mechanical wavefields and the electromagnetic fields is set to zero. Therefore, we have rederived the complete eigenvector sets for both the SH-TE and P-SV-TM propagation modes, thereby explicitly paying attention that the system remains stable when the coupling coefficient is set to zero. The details of this derivation go beyond the scope of this abstract. To correctly describe the reflections and transmissions that oc- cur at each interface, our algorithm makes use of an underlying global reflection scheme, originally derived for a three layer medium by Airy (1833). Making use of global reflection matrices has the benefit that explicit calculation of the scattering matrices at each individual interface is not required, leading to an effective numerical scheme. The global reflection matrices at each level are being calculated recursively, thereby using the already calculated reflection matrices at other levels. They describe the reflection and transmission of each of the wavetypes (fast P-wave, slow P-wave, SV-wave, $\mathrm{SH}$-wave, TM-field and TE-field) at the respective interface. The scheme starts with a local reflection coefficient at the top interface for upgoingto-downgoing wave reflections and at the bottom interface for downgoing-to-upgoing wave reflections. Internal multiples are included in the scheme, as well as top and bottom boundary reflections. Secondary waves are excluded. With secondary waves we mean waves that have been reflected back upwards or downwards, passing for the second time through the reference depth level where the down-up or up-down global reflection matrices, respectively, are defined, get reflected again, and then pass through the reference level for the third time.

At the top and bottom boundaries of the modeling domain, desired boundary conditions can be applied. Usually, the top interface makes use of free-surface conditions, whereas the bottom interface just describes the transition between a porous layer and a porous halfspace. However, different boundary conditions might be desired depending on what geometry one would like to investigate. For example, for fluid / porous media transitions, more complex boundary conditions apply. To completely simulate a typical seismoelectric laboratory set-up of a porous sample surrounded by water, the scheme must apply fluid / porous medium boundary conditions both at the top and the bottom interfaces of the model. At these interfaces, explicit boundary conditions must hold that describe the continuation or cancellation of certain field quantities correctly. In our scheme, we make use of the open-pore boundary conditions of Deresiewicz and Skalak (1963). Open-pore boundary conditions imply free flow of fluid across the fluid/porous medium interface (Denneman et al. (2002)). Alternatively, closed boundary conditions could be chosen. For the seismoelectric P-SV-TM system (denoted by the superscript V), and the SH-TE system (denoted by superscript $\mathrm{H}$ ), this effectively means (e.g. Pride and Haartsen (1996), Schakel and Smeulders (2010)):

$$
\begin{aligned}
\tilde{v}_{3}^{s}+\tilde{w}_{3} & =\tilde{v}_{3}^{\text {fluid }} \\
-\tilde{p} & =-\tilde{p}^{\text {fluid }} \\
\tilde{\tau}_{33}^{b}+\tilde{p} & =0 \\
\tilde{H}^{H, V} & =\tilde{H}^{H, V ; \text { fluid }} \\
\tilde{E}^{H, V} & =\tilde{E}^{H, V ; \text { fluid }} \\
\tilde{\tau}^{b ; H, V} & =0,
\end{aligned}
$$

or in words, continuity of combined vertical component particle velocity fields $\left(\tilde{v}_{3}\right)$ and vertical component filtration velocity fields $\left(\tilde{w}_{3}\right)$, (pore-)fluid pressure $(\tilde{p})$ and the horizontal component magnetic and electric fields of the SH-TE and P-SV-TM mode $\left(\tilde{H}^{H, V}\right.$ and $\tilde{E}^{H, V}$, respectively), opposed to the vanishing vertical component bulk stress $\tilde{\tau}_{33}^{b}$ and vanishing SH-TE and P-SV-TM horizontal component bulk stresses 
$\tilde{\tau}^{b ; H, V}$, respectively). The horizontal component SH-TE and PSV-TM particle velocity fields are 'free' since we have applied the boundary conditions to the bulk stress fields. The tilde sign denotes that we are in the horizontal wavenumber-frequency domain, the domain where the algorithm of 'ESSEMOD' calculates the majority of its time. We can write the fluid / porous medium boundary conditions as expressed in equations 1 for two-way wavefield quantities, in terms of the eigenvector matrices $\tilde{\mathbf{L}}^{H, V}$ multiplied with the one-way wavefield vector $\tilde{\mathbf{p}}^{H, V}$ as follows

$$
\tilde{\mathbf{L}}_{p . m . V}^{H, \mathbf{p}_{p . m .}^{H, V}}=\tilde{\mathbf{L}}_{\text {fluid }}^{H, V} \tilde{\mathbf{p}}_{\text {fluid }}^{H, V} .
$$

The boldface symbols indicate that we are dealing with matrices or vectors, p.m. stands for porous medium. The eigenvectors in $\tilde{\mathbf{L}}^{H, V}$ are the eigenvectors for the corresponding medium, for either the SH-TE or P-SV-TM propagation mode. The oneway wavefield vectors $\tilde{\mathbf{p}}^{H, V}$ contain the downgoing and upgoing wavefields for each of the wavetypes. In the fluid only (fast) P-waves and EM-fields can be sustained for the P-SVTM mode, and only EM-fields for the SH-TE mode. In other words, mechanical and electromagnetic fields are naturally decoupled. We have derived and flux-normalized the eigenvectors for the fluid media in a straightforward way.

In a fluid / porous medium / fluid configuration, reflection and transmission at both the top and bottom interface need to be correctly described and implemented using the above posed open-pore boundary conditions. Looking at the continuity of the wavefields, one can easily see which elements of the original porous media eigenvectors need to be combined (i.e. equations 1 and 3) to correctly describe the equality of equation 7. However, there is a difference between the top and bottom interface with respect to the upgoing-to-downgoing reflection matrix or the downgoing-to-upgoing reflection matrix. Or, differently formulated, the upgoing-to-downgoing reflection matrix is a local reflection matrix for the top interface and a global reflection matrix for the bottom interface, and vice versa for the downgoing-to-upgoing reflection matrix. The local reflection matrices can be derived quite straightforwardly. However, the global reflection matrices are more tedious to derive. For example, in order to find the global downgoing-to-upgoing reflection matrix at the fluid-side of the top-interface, correct boundary conditions must be applied while recursively updating the reflection matrix from porous medium to fluid. In other words, we need to express it in terms of the global reflection coefficient at the solid side of the interface. We can express the one-way upgoing wavefields in the solid in terms of the downgoing wavefields in the solid times the global reflection matrix at the solid side. Using these expressions and requiring the equality 7 to hold, we can reorganize the system such that we separate the unknown, outgoing one-way wavefields from the incoming one-way wavefields, assuming that the source is located in the top fluid halfspace. In this way, we can solve the system as

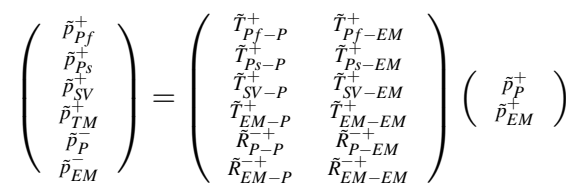

where $\tilde{R}^{-+}$are the global reflection matrix elements at the fluid side that we were after. To describe the transmission downwards from fluid to the porous medium, the global transmission matrix elements $\tilde{T}^{+}$can be used. In a similar way, the other local and global reflection and transmission matrices can be derived. These derived reflection and transmission matrices are then integrated in the existing numerical algorithm of 'ESSEMOD'.

\section{RESULTS}

Now that we have briefly explained the underlying theoretical fundaments of our numerical modeling code 'ESSEMOD, we can start with the actual validation of the code. We here want to focus on the validation of the underlying, adapted global reflection algorithm for typical laboratory configurations that contain fluid / porous medium / fluid transitions. To this end, we will compare the electromagnetic part of our code, with the results of an already existing electromagnetic layered Earth code 'EMMOD' (Hunziker et al., 2014).

We will model for $N t=2048$ amount of time samples, with a sampling step $d t=2.083333 \cdot 10^{-5} \mathrm{~s}$. The amount of radial frequencies $\omega$ is defined as $N_{\omega}=N t / 2+1$, and the radial frequency sampling rate $d_{\omega}=2 \pi /(N t \cdot d t) \mathrm{rad} \cdot \mathrm{s}^{-1}$. The wavelet is a first derivative of a Gaussian, with a peak frequency of 3 $\mathrm{kHz}$. We consider 51 receivers in both horizontal directions, with a spacing of $10 \mathrm{~m}$. All results represent the shot records at zero-offset for $x_{2}$, sliced along the $x_{1}$ direction. The positive $x_{3}$-axis is pointing downwards, indicating depth.

Let us consider a typical laboratory geometry with a porous medium (represented by a porous layer) in between two fluidhalfspaces. We will focus on three different experiments. Note that the scale is not representing a true laboratory scale. Firstly, a full transmission experiment where we place the electric current source (in the horizontal $x_{1}$ direction) in the upper fluid halfspace at $-40 \mathrm{~m}$ depth, and the horizontal $x_{1}$ electric field component receivers in the lower fluid halfspace at $1200 \mathrm{~m}$ depth. The fluid / porous medium and porous medium / fluid interfaces are located at $0 \mathrm{~m}$ and $1000 \mathrm{~m}$ depth, respectively. As can be clearly observed in Figure 1 a, the modeled results of 'ESSEMOD' and 'EMMOD' perfectly match in both amplitude and phase. This shows that the full transmission is correctly modeled in 'ESSEMOD'. Often in seismoelectric wave propagation laboratory tests, the receivers / electrodes are placed in the solid, whereas the source is an acoustic pressure source located in the fluid. To model these specific configurations, we again consider a source at $-40 \mathrm{~m}$ in the upper fluid halfspace, but now the receivers are being placed in the porous medium, at $600 \mathrm{~m}$. The rest of the geometry remains the same. The results are presented in Figure 1b. It can be clearly observed that the numerical reflection algorithm of 'ESSEMOD' can also handle these specific geometries correctly. Thirdly, we will consider a pure reflection geometry, i.e. source and receivers both placed in the upper fluid halfspace. To investigate whether the recursive updating of the global reflection matrices works properly, we have added another porous layer to the porous medium. The interface separating these two porous layers is positioned at $700 \mathrm{~m}$. We now place the source at -400 $\mathrm{m}$ and the receivers at $-30 \mathrm{~m}$. The rest of the geometry stays identical to the previous model runs. Figure 1c clearly shows that the results of 'ESSEMOD' and 'EMMOD' also perfectly 


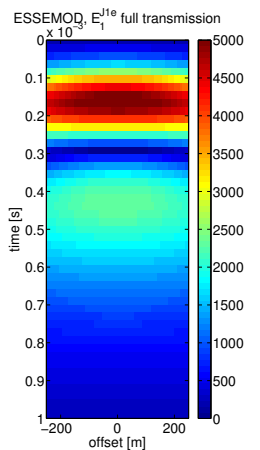

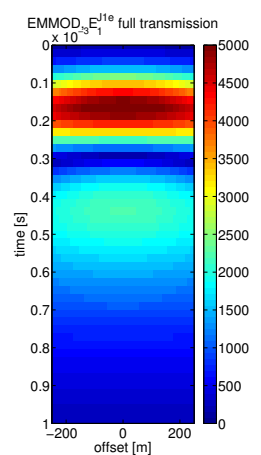

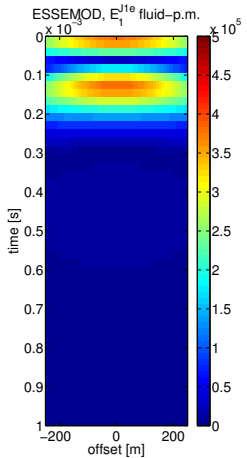

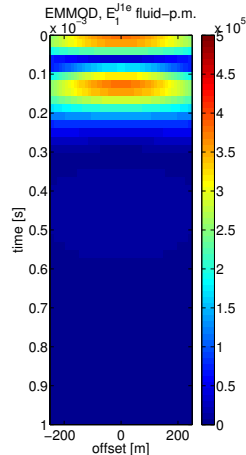

(b)

Figure 1: Comparison between 'ESSEMOD' (left panels) and 'EMMOD
in the fluid, receivers in the porous medium. (c) Reflection experiment.

match in both phase and amplitude for this reflection experiment, indicating correct recursive calculation of the global reflection matrices. As a final experiment, we will simulate seis-
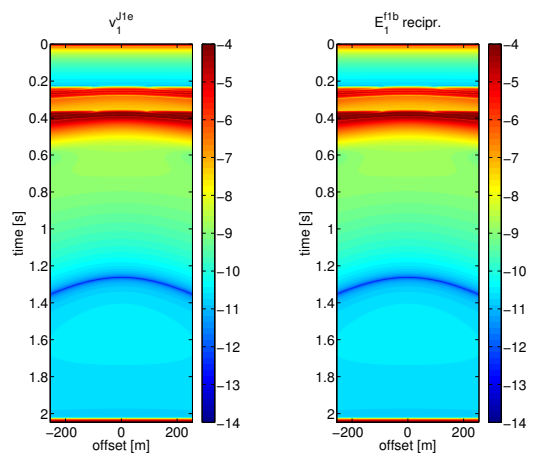

Figure 2: (left) $v_{1}^{J_{1}^{e}}$. (right) The reciprocal experiment: $E_{1}^{f_{1}^{b}}$ moelectric wave propagation in a fluid / porous medium configuration, with the interface located at $x_{3}=0 \mathrm{~m}$. We have removed the lower fluid halfspace, to avoid complex shot records due to multiple seismic reverberations in the porous layer. We will test the algorithm by carrying out a reciprocity test. We will first model a horizontal particle velocity field in the $x_{1}$ direction, registered in the porous halfspace at $x_{3}=800 \mathrm{~m}$, due to a horizontal $x_{1}$ oriented electric current source placed in the fluid halfspace at $x_{3}=-100 \mathrm{~m}$. For the reciprocal test, we turn every receiver at $x_{3}=800 \mathrm{~m}$ into a source and place only one receiver at $x_{3}=-100 \mathrm{~m}$. The seismoelectric reciprocal field of $v_{1}^{J 1 e}$ is $E_{1}^{f 1 b}$, the horizontal component electric field in the $x_{1}$ direction due to a seismic dipole bulk force source in the same direction. The results in Figure 2 show that also the reciprocal test for seismoelectric wave propagation in fluid / porous medium configurations matches in both phase and amplitude. We expect two interface response fields, related to the seismic $\mathrm{P}$ - and $\mathrm{S}$-wave propagation in the porous medium. The fields arrive at traveltimes corresponding to the one-way path of the mechanical waves in the porous medium.

\section{DISCUSSION AND CONCLUSIONS}

As we have shown in the previous section, the results of the validation tests indicate that 'ESSEMOD' is correctly modeling the electromagnetic parts of the seismoelectric wave propa- gation in horizontally layered media with fluid / porous medium / fluid transitions. The results show a perfect match in both phase and amplitude for full transmission and pure reflection experiments as well as for a combination of both. By focusing on the electromagnetic parts only (by choosing the seismoelectric coupling coefficient equal to zero), we were able to effectively test the recursive global reflection algorithm of 'ESSEMOD' in configurations that resemble typical seismoelectric laboratory configurations. In addition, the seismoelectric reciprocity test in a configuration of a fluid halfspace overlying a porous medium halfspace, shows that also the coupled poroelastic and electromagnetic waves are modeled consistently and yield the expected results.

However, not all aspects of the modeling code have been validated here. Previously, the amplitudes and phase have been successfully validated for the full seismoelectric theory in homogeneous space geometries (Grobbe and Slob (2013)). This gives us some control on the modeling results, but it does not validate the underlying reflection mechanism of ESSEMOD. In an attempt to validate this reflection scheme for typical seismoelectric laboratory configurations with fluid / porous medium transitions, we have here compared the electromagnetic part of the theory with an existing layered Earth code for electromagnetic fields. The poroelasticity part of the seismoelectric theory has not yet been compared with any other existing layered Earth codes. Nevertheless, the reflection scheme is identical for the electromagnetic part and the full seismoelectric theory. In addition, the seismoelectric reciprocity test shows consistent modeling of seismoelectric wave phenomena in configurations with fluid / porous medium transitions.

In order to be able to really validate the numerical modeling and its underlying theory, we need to compare ESSEMOD with physical laboratory experiments. The algorithm shows to be working correctly for typical laboratory geometries, thereby clearing the way to comparisons of this type.

\section{ACKNOWLEDGMENTS}

The research was funded as a Shell-FOM (Fundamental Research on Matter) project within the research program "Innovative physics for oil and gas". The authors are grateful to Jan Thorbecke for his help coding ESSEMOD. 
http://dx.doi.org/10.1190/segam2014-0689.1

\section{EDITED REFERENCES}

Note: This reference list is a copy-edited version of the reference list submitted by the author. Reference lists for the 2014 SEG Technical Program Expanded Abstracts have been copy edited so that references provided with the online metadata for each paper will achieve a high degree of linking to cited sources that appear on the Web.

\section{REFERENCES}

Airy, G., 1833, On the phenomena of Newton's rings when formed between two transparent substances of different refractive powers: The London and Edinburgh Philosophical Magazine and Journal of Science, 2, 20-30.

Denneman, A., G. Drijkoningen, D. Smeulders, and K. Wapenaar, 2002, Reflection and transmission of waves at a fluid/porousmedium interface: Geophysics, 67, 282-291.

Deresiewicz, H., and R. Skalak, 1963, On uniqueness in dynamic poroelasticity: Bulletin of the Seismological Society of America, 53, 783-788.

Garambois, S., and M. Dietrich, 2002, Full-waveform numerical simulations of seismoelectromagnetic wave conversions in fluid-saturated stratified porous media : Journal of Geophysical Research, 107, no. B7.

Grobbe, N., and E. Slob, 2013, Validation of an electroseismic and seismoelectric modeling code, for layered earth models, by the explicit homogeneous space solutions: Presented at the $83^{\text {rd }}$ Annual International Meeting, SEG.

Grobbe, N., J. Thorbecke, and E. Slob, 2012, Essemod electroseismic and seismoelectric flux-normalized modeling for horizontally layered, radially symmetric configurations: Geophysical Research Abstracts EGU 2012, 14, 10011.

Haartsen, M., and S. Pride, 1997, Electroseismic waves from point sources in layered media : Journal of Geophysical Research, 102, no. B11, 24745-24769.

Hunziker, J., J. Thorbecke, and E. Slob, 2014, The electromagnetic response in a layered VTI medium: A new look at an old problem: Geophysics, in press.

Pride, S., 1994, Governing equations for the coupled electromagnetics and acoustics of porous media: Physical Review B: Condensed Matter and Materials Physics, 50, no. 21, 15678-15696, http://dx.doi.org/10.1103/PhysRevB.50.15678. PubMed

Pride, S., and M. Haartsen, 1996, Electroseismic wave properties: The Journal of the Acoustical Society of America, 100, no. 3, 1301-1315, http://dx.doi.org/10.1121/1.416018.

Revil, A., N. Linde, A. Cerepi, D. Jougnot, S. Matthäi, and S. Finsterle, 2007, Electrokinetic coupling in unsaturated porous media : Journal of Colloid and Interface Science, 313, no. 1, 315-327, http://dx.doi.org/10.1016/j.jcis.2007.03.037. PubMed

Schakel, M., and D. Smeulders, 2010, Seismoelectric reflection and transmission at a fluid/porousmedium interface: The Journal of the Acoustical Society of America, 127, no. 1, 13-21, http://dx.doi.org/10.1121/1.3263613. PubMed

Schakel, M., D. Smeulders, E. Slob, and H. Heller, 2011, Laboratory measurements and theoretical modeling of seismoelectric interface response and coseismic wavefields: Journal of Applied Physics, 109, 074903-1 - 074903-5. 
Schoemaker, F., N. Grobbe, M. Schakel, S. de Ridder, E. Slob, and D. Smeulders, 2012, Experimental validation of the electrokinetic theory and development of seismoe lectric interferometry by crosscorrelation: International Journal of Geophysics, http://dx.doi.org/10.1155/2012/514242.

Smeulders, D., N. Grobbe, H. Heller, and M. Schakel, 2014, Seismoelectric conversion for the detection of porous medium interfaces containing water and oil: Vadose Zone Journal, in press.

Thompson, A., and G. Gist, 1993, Geophysical applications of electrokinetic conversion: The Leading Edge, 12, 1169-1173, http://dx.doi.org/10.1190/1.1436931.

Ursin, B., 1983, Review of elastic and electromagnetic wave propagation in horizontally layered media : Geophysics, 48, 1063-1081, http://dx.doi.org/10.1190/1.1441529.

Zhu, Z., M. Haartsen, and M. Toksöz, 2000, Experimental studies of seismoelectric conversions in fluidsaturated porous media: Journal of Geophysical Research, 105, B12, 28055-28064, http://dx.doi.org/10.1029/2000JB900341.

Zhu, Z., and M. Toksöz, 2005, Seismoelectric and seismomagnetic measurements in fractured borehole models: Geophysics, 70, no. 4, F45-F51. 